Léglise, I. (2017), « Multilinguisme et hétérogénéité des pratiques langagières. Nouveaux chantiers et enjeux du Global South ", Langage et Société n¹40-141, 251-266.

LÉGLISE Isabelle

CNRS, UMR 8202 SeDyL

isabelle.leglise@cnrs.fr

\title{
Multilinguisme et hétérogénéité des pratiques langagières. Nouveaux chantiers et enjeux du Global South ${ }^{1}$
}

\author{
Cet article présente d'abord quelques travaux récents dans l'étude du \\ multilinguisme et de ses conséquences - linguistiques et sociales - en \\ insistant sur des aspects méthodologiques. Il se fonde sur des exemples \\ provenant de terrains situés dans les suds et sur l'analyse de pratiques \\ langagières hétérogènes qui obligent à revoir les catégories descriptives \\ traditionnelles. \\ Il présente ensuite des domaines - comme autant de chantiers à explorer - \\ dans lesquels les enjeux du Global South sont particulièrement criants : \\ dans le domaine de la citoyenneté linguistique (plutôt que des droits \\ linguistiques), dans le domaine de l'éducation plurilingue et \\ interculturelle et dans le domaine de la santé.
}

This paper first presents recent works in the study of multilingualism and its linguistic and social consequences, focusing on methodology. It is based on Southern case studies and heterogeneous language practices which invalidate traditional descriptive categories. It focuses then on domains with striking socio-political issues of the Global South: linguistic citizenship rather than linguistic rights, multilingual and multicultural Education and Health.

Mots clés : multilinguisme, Suds, postcolonial, éducation plurilingue, justice sociale, santé

\section{Introduction}

Linguistes et anthropologues ont sillonné les terrains lointains ces deux derniers siècles. En même temps que leurs disciplines académiques s'établissaient en Occident, ils participaient à la définition et à la construction des langues et des cultures (Gal et Irvine 1995) dans un ailleurs, les Suds (dont les qualificatifs empruntent généralement à l'exotisme et à l'infériorité : ex-colonies, Tiers Monde etc.), ayant servi de réservoir de "données brutes » (Comaroff et Comaroff 2012 : 113) pour des théorisations occidentales. Or, les Suds semblent préfigurer ce qui arrive dans tout pays en temps de récession ou de crise économique, tant dans le domaine des mouvements de population, de l'exclusion sociale, des émeutes que de la racialisation des rapports sociaux (Comaroff et Comaroff 2012). Si cette présentation peut faire croire à une division géographique du monde, il faut plutôt comprendre les Suds - ou le Global South - comme « une métaphore de la souffrance humaine causée par le capitalisme et le colonialisme à l'échelle mondiale, et de la résistance visant à la surmonter ou à l'atténuer » (Santos 2011 : 39), que ce soit au Nord, comme dans les différents Suds.

\footnotetext{
${ }^{1}$ Ce texte doit beaucoup aux lectures et échanges lors du séminaire « Pratiques langagières : terrains, méthodes, théories » avec les intervenants invités, les doctorants et ma collègue V. Muni Toke.
} 
L’une des caractéristiques linguistiques majeures de ce Global South est le multilinguisme des territoires et le plurilinguisme de la population. D’un point de vue linguistique, cela se traduit par des pratiques langagières hétérogènes pour lesquelles il a fallu adapter notre appareillage conceptuel. D'un point de vue social, ce multilinguisme, conçu comme la coexistence, dans un contexte ou sur un territoire donné, de langues, pratiques et variétés, aux statuts économiques et symboliques variés, se traduit par une répartition inégale des ressources au sein de la population. Cela pose en particulier des questions d'équité et d'accès aux soins ou à l'éducation de pans entiers de la population.

Travailler sur ces terrains dans une perspective postcoloniale nécessite deux décentrements : d'une part l'abandon ou l'évolution des catégories descriptives traditionnelles, d'autre part un autre rapport au "terrain » et à l'expertise scientifique avec un recentrement sur les perceptions et points de vue endogènes pour faire entendre leurs voix. Les théorisations occidentales ont en effet confiné au silence l'expérience locale des populations et les théorisations alternatives. Un effet d'effacement massif caractérise ainsi les Théories du Sud (Connell 2007) laissées à la périphérie du système de production de connaissances. S’intéresser à présent au Global South nécessite de «révéler les silences épistémiques » des épistémologies occidentales en affirmant «les droits épistémiques de ceux qui sont racialement dévalués » (Mignolo 2009, 4). Nous verrons comment des formes de visibilisation - de ces savoirs et de ces pratiques - sont possibles.

\section{Du contact de langues aux pratiques langagières hétérogènes}

Alors que les terrains ont toujours été multilingues (qu'il s’agisse de terrains situés dans des colonies ou dans des campagnes éloignées), l'entreprise massive de description des langues et dialectes qui s'est développée à partir du XIXe siècle reposait sur la focalisation sur une langue, c'est-à-dire en faisant abstraction du contexte multilingue. On sait que cette entreprise, prise dans l'orientalisme ambiant, a contribué à construire à la fois ces langues exotiques comme "langues » et comme «objets exotiques » en même temps que les EtatsNations européens émergeaient dans un processus de différenciation politique (Gal et Irvine 1995). Les travaux critiques sur la linguistique coloniale ont bien montré également comment, du XVIe au XXe siècle, le pouvoir naturalisant des descriptions linguistiques et l'imposition de catégories coloniales comme modèles d'identités ethnoculturelles (Errington 2001) ont renforcé les logiques territoriales des pouvoirs coloniaux et participé par exemple à l' « invention » de l'Afrique et des langues africaines (Mudimbe 1988 ; Makoni et Pennycook 2007).

D'autre part, la focalisation sur les langues (en fait à chaque fois sur une seule) revenait également à faire abstraction du contexte social ou socio-culturel tout en assignant aux locuteurs l'identité culturelle sensée être véhiculée par la langue 'maternelle', 'locale' ou 'indigène' qu'ils parlaient. Ce que Gal et Irvine appellent «l'utilisation persistante de la langue comme synecdoque de communauté » (ibid : 968) s'appuyant sur la corrélation non questionnée «une langue égale une culture " s'est traduit par un construit idéologique particulièrement prégnant : chacun a fait comme si les langues étaient parlées au sein de communautés monolingues et monoculturelles. Ce construit, bien que critiqué il y a déjà cinquante ans car il laisse de côté les questions de variation, de multilinguisme et de construction sociale du langage (Hymes 1967), est toujours le soubassement d'un certain nombre d'approches, de la description des langues à la définition de politiques linguistiques éducatives par exemple.

De la même manière, un certain nombre de travaux sur le contact de langues et de cultures pose également l'existence de communautés linguistiques et culturelles isolées pour pouvoir 
s’intéresser ensuite aux conséquences du contact entre ces dernières. Ces contacts sont de fait considérés comme des phénomènes marginaux intervenant lors de complexifications de situations 'normales' monolingues (Nicolai 2007). Ainsi, les travaux sur le codeswitching nécessitent la séparation préalable des 'codes' concernés avant de montrer comment ils alternent. Les différentes approches se fondent toutes sur l'identification de langues en contact dans des corpus généralement bilingues, parfois plurilingues (Léglise à paraître).

Dans le contexte du Global South, les concepts de langues, contacts de langues ou codeswitching paraissent ainsi particulièrement inadéquats à la fois en raison de l'histoire coloniale dont ils sont chargés et en raison de la réalité multiforme des pratiques langagières observées sur le terrain. Les travaux postcoloniaux ont ajouté leur part dans la désinvention du mythe des langues (Makoni et Pennycook 2007) en montrant comment, de l'avis même des locuteurs ou de chercheurs issus des terrains concernés, les outils conceptuels forgés - et notamment le nom des langues - ne renvoyaient pas à leurs propres perceptions. D’autre part, pour rendre compte des situations de superdiversité rencontrées (Vertovec 2007) et des pratiques marquées par le multilinguisme et par ce que certains ont nommé l'hybridité, de nombreux chercheurs ont tenté de catégoriser différemment leurs objets d'étude ces dernières années : phénomènes de crossing (Rampton 2005), répertoires linguistiques superdivers ou tronqués (Blommaert et Backus 2011 ; Blommaert et Rampton 2011), polylanguaging (Jørgensen et al. 2011), translanguaging (García 2009b ; Garcia et Wei 2013), etc. (cf. Léglise à paraître pour une revue de la littérature). Les différents termes ont tous fait l'objet de critiques, même si celui de translanguaging semble actuellement le plus utilisé. La notion d'hybridité ou d'hybridisation a été critiquée par exemple car elle ne sort pas de l'identification d'entités discrètes et repose sur l'hypothèse qu'il existe deux codes ou entités séparées qui sont ensuite combinées (Makoni 2011, 683).

Cette insistance sur les pratiques plutôt que sur les langues n'est toutefois pas nouvelle en sociolinguistique. Dans cette quête du terme idoine pour décrire les pratiques auxquelles nous sommes confrontés, le terme de "pratiques langagières hétérogènes » m'a paru le plus adéquat d'une part parce que l'adjectif langagières plutôt que linguistiques permet d'éviter la nomination d'entités discrètes, d'autre part parce qu'il permet d'englober les phénomènes de variation et de pluri-accentuation. Les premiers travaux ayant proposé le terme de pratiques langagières (Boutet, Fiala et Simonin-Grumbach 1976), ont révélé l’hétérogénéité sociale de pratiques langagières monolingues et leur pluri-accentuation. Les pratiques langagières étudiées dans le cadre du Global South sont hétérogènes au sens où elles sont non seulement pluri-accentuées mais aussi plurilingues. Elles sont produites par des locuteurs plurilingues aux compétences, ressources et répertoires linguistiques variés. Au lieu de décrire ces pratiques comme du codeswitching ou du code-mixing, on y observe l'utilisation de ressources langagières par les acteurs sociaux - utilisation qui a pu être décrite comme le «bricolage linguistique » (Lüdi 1994 ; Mondada 2012) par lequel ces derniers utilisent un ensemble de ressources existantes - parfois anciennes - pour construire de nouvelles significations.

Ces pratiques peuvent indexer et participer à construire, par exemple, une identité urbaine et moderne chez les jeunes hommes businenge en Guyane et au Surinam qui puisent dans leurs ressources pour insérer des éléments lexicaux et structuraux de langues internationales (français, anglais, néerlandais) aux variétés créoles qu’ils parlent quotidiennement, dans d'autres contextes, et dont la pratique unique pourrait renvoyer à une ruralité dont ils veulent s'éloigner. La nomination mouvante de ces pratiques relève, également, de points de vue et d’idéologies locales et globales (Migge et Léglise 2013). 


\section{De l'enseignement des langues maternelles à la prise en compte des pratiques langagières hétérogènes}

Pris entre décolonisation et globalisation, les systèmes éducatifs constituent l'un des champs de bataille du Global South. Généralement fondés durant la période coloniale, ces derniers, de l'Afrique à l'Amérique latine, sont conçus sur des modèles européens et, à ce jour, continuent d'implémenter à plus ou moins grande échelle beaucoup de politiques linguistiques et culturelles héritées de cette époque - ce qui perpétue les idéologies coloniales et les hiérarchies de langues et de variétés (Migge et Léglise 2007). Par exemple, dans la plupart des sociétés créoles, et en dépit de changements sociaux majeurs ces cinquante dernières années, les systèmes éducatifs ont peu évolué dans l'ensemble. Alors que les élèves ne sont plus punis pour l'utilisation de leurs langues pidgins ou créoles en classe, ce qui fut longtemps le cas, peu de ces langues sont officiellement reconnues comme medium d'enseignement, autrement que comme mesures transitionnelles pour faciliter l'acquisition de langues officielles et européennes et souvent uniquement à l'oral (Migge, Léglise et Bartens 2010). Comme nous l'avons montré, cette utilisation des langues pidgins et créoles seulement à l'oral entre dans un cercle vicieux, car elle a pour corollaire l'absence de développement d'une littératie et d’une ingénierie de la langue écrite dues à la croyance selon laquelle les créoles ne sont pas appropriés à l'écrit qui, en retour, renforce cette même croyance d'une inadéquation des créoles à l'écrit chez les enseignants et les politiques parfois issus des mêmes « communautés linguistiques ».

Ainsi, alors même que les travaux montrent qu'enseigner à des enfants dans une langue qu'ils ne comprennent pas mène à l'échec éducatif (García 2009b) ou que l'utilisation des langues de première socialisation, comme médium d'enseignement et comme entrée dans la littératie, contribue fortement à réduire les chiffres de l'échec scolaire (Cummins 2009), l'intégration dans les systèmes éducatifs des langues et variétés minorisées demeure l'un des domaines importants d'étude - et d'enjeux pour la recherche à venir. Les problématiques ont cependant progressivement évolué. Historiquement impliqués auprès des communautés dont ils décrivaient des langues, les linguistes ont souvent été sollicités et ont participé à la grammatisation des langues minoritaires en vue de leur implémentation dans les systèmes éducatifs selon deux modèles d'éducation multilingue basée sur les langues maternelles (Mother Tongue-based Multilingual Education) pour plus de justice sociale (SkutnabbKangas et al. 2009). Qu'il s'agisse de maintenir les langues en visant un enseignement bilingue langue minoritaire-langue majoritaire ou de programmes immersifs, les travaux ont montré l'importance de s'appuyer sur l'expérience locale pour que les solutions envisagées fonctionnent. Une forte implication locale n'est pas seulement importante pour produire des matériaux didactiques authentiques : leur authenticité les rend également disponibles pour un lectorat plus important et aide à restaurer chez les locuteurs minorisés un sentiment de possession (ownership) des langues et variétés disponibles (Stroud 2001) qui peut contribuer à leur empowerment.

Si on prend l'exemple de la Guyane française, région d'Outre-mer où les dispositifs de l'Education nationale pensés au départ pour la métropole s'appliquent, longtemps l'écart entre les langues parlées en famille et la langue de l'école a été perçu comme la cause des problèmes éducatifs et du taux important de sortie du système sans diplôme. Il est vrai que, malgré l'entreprise de francisation lancée en 1946 lors du changement de statut de colonie à département, et malgré l'obligation de scolarisation datant de 1970 (Puren 2007, 284-sv.), plus de 2/3 des enfants scolarisés n’y parlent pas français avant d'entrer à l'école. Le français est en effet très peu présent dans certains contextes et zones géographiques où d'autres formes de communication (autres langues ou pratiques langagières hétérogènes) servent de 
véhiculaires. Depuis une quarantaine d'années, linguistes et anthropologues ont à la fois critiqué des «écoles non adaptées » (Grenand 1982) et milité, avec un certain succès, pour l’introduction des langues maternelles dans le système éducatif (Launey 1999), parallèlement aux mouvements militants créoles (Prudent et Schnepel 1993) et aux côtés des leaders amérindiens ou noir-marrons ( $c f$. l'extrait suivant du discours fondateur de la revendication amérindienne (Tiouka 1984) sur la question de la souveraineté :

\begin{abstract}
Aujourd'hui, nous pensons que la reconnaissance de cette souveraineté doit être à la base de la redéfinition devenue urgente et nécessaire de nos rapports avec la société dominante. Cette redéfinition doit être l'occasion pour nous d'établir notre contrôle sur les institutions et les processus de décision qui nous touchent le plus directement dans les domaines du développement économique, de l'éducation, de la santé et des services sociaux, ainsi que de l'organisation politique locale et régionale, etc. En un mot, forts de traditions millénaires, nous voulons rétablir et renforcer nos valeurs culturelles propres dans les domaines institutionnels nous concernant.

La référence à nos valeurs traditionnelles indique clairement que nous refusons de considérer comme valable l'option de l'assimilation progressive à la société dominante qui est insidieusement en cours et qui est encouragée directement ou indirectement par tous les agents politiques, administratifs, économiques, faisant affaire avec nous.

Nous voulons demeurer Amérindiens et conserver notre langue, notre culture, nos institutions propres.
\end{abstract}

En raison des cadres imposés par l’Education nationale, ces efforts conjugués n’ont permis, et à la suite de nombreux rebondissements (Goury et al. 2005), de développer que des programmes d'enseignement bilingue transitionnels (à raison de quelques heures par semaine puis plus récemment à parité horaire ${ }^{2}$ ). L’objectif des programmes mis en place demeure la maîtrise du français pour permettre, au bout de quelques années, un enseignement uniquement en français. Ils s’appuient ainsi sur une vision du bilinguisme qui a été qualifié de " soustractif » et que l'on peut schématiser, en suivant García (2009b), comme L1+L2-L1 = L2. Toutefois, malgré l'existence actuelle de ces programmes, une analyse des discours des enseignants et des inspecteurs montre que, même lorsqu'ils acceptent l'idée d'introduire certaines langues dans les classes, ce qui n’est pas donné, ils insistent néanmoins sur l'obligatoire séparation des langues, incarnée la plupart du temps par des personnes différentes (une personne, une langue), des horaires spécialement dédiés et des espaces différentiés - et hiérarchisés - dans les classes : le fond de la classe pour les objets folkloriques renvoyant à l'espace créole ou amérindien et le tableau pour l'espace français (Alby et Léglise 2014). Nous avons pu montrer que le système éducatif en Guyane, basé sur une idéologie nationale promouvant le monolinguisme et la francisation, ignore de fait la réalité plurilingue quotidienne de la population scolarisée, et que ce n'est pas seulement l'écart entre langue de l'école et langue de la maison qui est problématique, mais c'est l'écart entre, d'une part, une forme de ségrégation des langues liée à des normes monolingues exogènes et, d'autre part, l'expérience langagière vécue - au sens de Busch (2015) quotidienne, locale, hétérogène et plurilingue. On sait que si, au sein de la langue de scolarisation, on ne laisse pas de place au «languaging des élèves, et si l'enseignant ne maximise pas la communication en utilisant les pratiques langagières des élèves, on peut être sûr qu'un échec dans la communication et l'éducation va se produire » (García 2009b, 152).

\footnotetext{
${ }^{2}$ D'abord mis en place de manière expérimentale, un dispositif bilingue d'enseignement français-créole à parité horaire existe depuis 2008. La possibilité d'étendre ce dispositif aux autres langues créoles et amérindiennes semble ouverte depuis la modification de 2013 du Code de l'éducation qui spécifie désormais «Dans les académies d'outre-mer, des approches pédagogiques spécifiques sont prévues dans l'enseignement de l'expression orale ou écrite et de la lecture au profit des élèves issus de milieux principalement créolophone ou amérindien. " (Article L321-4 Modifié par LOI n²013-595 du 8 juillet 2013 - art. 46). L’implémentation réelle de ce dispositif à d'autres langues en Guyane demeure, à ce jour, incertaine. (Cf. la question posée au gouvernement par la députée C. Berthelot sur le " bilinguisme dans les écoles primaires de Guyane » en juin 2016, https://www.nosdeputes.fr/14/seance/6794\#table_16438)
} 
Sans doute pour éviter cet échec permanent, observe-t-on, dans les classes, que les élèves - et parfois les enseignants qui sont alors en porte-à-faux avec leur hiérarchie - utilisent leur potentiel plurilingue pour maximiser leurs chances de communiquer et d'apprendre (García 2009a). Ainsi, malgré l’idéologie de séparation des langues dans les discours institutionnels, la réalité plurilingue des élèves - et des enseignants - fait surface dans les interactions de classe, au sein de pratiques langagières hétérogènes, et montre, localement, un certain degré d’agentivité à la fois des élèves et des enseignants (Alby et Léglise 2016).

Ainsi, c'est non seulement l'intégration des langues et variétés minorisées dans les systèmes éducatifs qui demeure un enjeu pour la recherche à venir dans les Suds mais également une meilleure connaissance, prise en compte - et intégration didactique - de l'expérience langagière intime et des pratiques langagières hétérogènes des élèves, de la population, et des enseignants en tant qu'acteurs.

\section{Visibiliser les variétés minorisées et les pratiques littératiques non reconnues}

Une façon de renverser les stigmas affectant des groupes minorisés est ainsi de visibiliser des variétés minorisées et de développer une conscience critique sur les variétés normées et les pratiques littératiques reconnues. Alim (2011) a proposé le terme de ill-literacies pour renvoyer aux pratiques littératiques hybrides et transculturelles du hip hop, qu'elles soient locales ou globales. En utilisant le préfixe ill-, il insiste non pas sur le " défaut de littératie » qui est sous-entendu par un concept comme illéttrisme - notion sociopolitiquement construite et définie par rapport à certaines formes dominantes de littératie - mais sur la présence de compétences littératiques spécifiques au sein de certains groupes minorisés que ce soit dans certains quartiers Nord-Américains ou en Afrique. Ces pratiques littératiques se caractérisent par leur aspect créatif et contre-hégémonique et doivent être intimes, vécues et libératoires (Literacy must be Intimate, Lived and Liberatory) (ibid, 123). Ces pratiques hétérogènes permettent aux jeunes de remettre en cause non seulement les relations de pouvoir et d'identification entre langues et cultures mais aussi d'exprimer leurs expériences intimes comme un potentiel de transformation sociale plus globale. Les exemples sont nombreux de l'utilisation des pratiques littératiques de hip hop à des fins de contestation et d'articulation de multiples identités. Omoniyi (2006) par exemple montre comment les jeunes nigérians, utilisent le hip hop et les pratiques langagières hétérogènes qui y sont liées en ayant recours à différentes langues et variétés locales (yoruba, igbo), à des formes locales d'anglais-américain mais surtout au pidgin du Nigéria. Ces pratiques littératiques leur permettent d'explorer et de réinventer des identités multiples et de contester l’idéologie dominante héritée de la période coloniale qui impose l'anglais à la fois comme véhiculaire et comme langue officielle commune à tous, alors que leurs pratiques s’appuient surtout sur le pidgin du Nigéria comme forme véhiculaire.

Des pédagogies critiques employant le hip hop (Critical Hip Hop Language Pedagogies) se sont développées dans le but de développer la conscience métalittératique chez les étudiants qui s'appuie sur la prise de conscience de sa propre position dans le monde et de ce qu'on peut y faire (Alim 2004). Le but des travaux sur la littératie dans le Global South est de rendre les étudiants capables de «critiquer les pratiques hégémoniques qui ont formé leurs expériences et leurs perceptions pour les délivrer des idéologies, des structures et des pratiques dominantes » (Morrell et Duncan-Andrade 2004, 250). Le travail engagé vise ainsi à définir son être littératique comme « être présent, et actif dans la lutte pour réclamer sa propre voix, son histoire et son futur " (ibid, 249). En visibilisant des formes habituellement invisibles, ou "langues de couleur », les auteurs espèrent ainsi examiner comment les 
enseignants et éducateurs par exemple, en inculquant aux locuteurs de variétés hétérogènes les normes de la classe moyenne blanche, imposent les façons de parler et de voir le monde des Blancs (Alim 2011 : 133). Il s’agit ici de tenter de subvertir, les mécanismes de reproduction, de naturalisation et de légitimation par l'école (Bourdieu et Passeron 1970, Heller et Martin Jones 2001), d’inégalités sociales et raciales tout comme un travail précis sur les interactions en classe peut permettre de montrer la construction des inégalités sociales dans les classes (Rojo 2015) et la construction de ce qu’est le savoir légitime.

Dans ce domaine, il reste encore beaucoup à faire pour visibiliser l'invisible (Kerfoot et Hyltenstam 2017) de populations renvoyées au silence ou marginalisées - comme les réfugiés ou les migrants par exemple dans les pays occidentaux, ou à rendre visibles et légitimes différentes formes de savoir.

\section{Pratiques langagières à l'hôpital et rapport au savoir}

L'accueil des populations par les services publics est un excellent observatoire de la prise en compte ou non, par L'Etat et les différentes institutions concernées, des langues et variétés minorisées. C'est un lieu de visibilisation ou au contraire de réduction au silence d'un multilinguisme qui va souvent de pair avec la négation des connaissances et de l'expertise des usagers - en particulier celles des populations marginalisées. Le domaine de la santé est particulièrement éclairant. A l'hôpital, un certain nombre de stratégies (de traduction, médiation ou apprentissage de la langue de l'autre) sont possibles pour éviter les conséquences d'une communication exolingue dorénavant bien identifiées tant dans les phases de diagnostic, les retards d'admissions, l'information des patients que dans la prise en compte de leur témoignage, de leur adhésion au traitement, de leur participation à la prise de décision, leur satisfaction etc. (Bischoff 2003). L'existence de manuels plurilingues d'aide au diagnostic, par exemple, n'est pas forcément une sinécure. Collins et Slembrouck (2006) ont montré comment, dans une clinique en Belgique, ces manuels devaient permettre aux médecins de (tenter de) lire les traductions dans différentes langues aux patients mais pas de prendre en compte leurs réponses - ce qui montre bien d'une part que le patient idéal est construit comme silencieux et que d'autre part, il n'est pas certain que ce type de pratiques littératiques facilitent la communication.

En Guyane, j'ai pu montrer que la prise en compte de la diversité linguistique et culturelle des patients ne faisait pas partie des politiques linguistiques poursuivies à l'hôpital, elle reposait uniquement sur le bon vouloir des soignants, pouvait dans le meilleur des cas permettre la transmission minimale d'injonctions de l'équipe médicale mais reposait la plupart du temps sur des assignations d'identité trahissant une racialisation des rapports sociaux (Léglise 2007). En tant que population doublement fragilisée, les patients, locuteurs de langues et variétés minorisées, sont de fait réduits au silence (Muni Toke 2017) et rares sont les situations où ils peuvent faire entendre leur voix. Le maintien de conversations en parallèle, dans une chambre d’hôpital, entre la famille d'une patiente et l'équipe médicale, vécu comme profondément impoli, où des frontières hermétiques (de langues et de savoirs) sont construites dans l'interaction, peut traduire justement, face à un savoir médical délivré en français, un moment de revendication d'une légitimité épistémique à s'occuper de leur mère, alitée et livrée à la médecine occidentale (Léglise à paraître). Ces moments de malentendus, d'absence de rencontre dans la communication, d'expression d'une forme de résistance par des pratiques langagières hétérogènes, sont précieux. Kanj et Mitic (2009) montrent justement comment la participation du patient et le fait de faire entendre sa voix sont importants. Pour eux, une compétence littératique en santé est atteinte lorsque les individus comprennent leurs droits en 
tant que patients, qu'ils savent se repérer dans le système de santé, prendre des décisions en étant informés et les verbaliser. Il s'agit d’un puissant outil d’émancipation et les différentes langues peuvent permettre de favoriser cette pleine participation à la prise de décision et l'émergence d’une citoyenneté de santé (Thutloa et Stroud 2013).

\section{Conclusion}

Alors que les tendances mondiales dominantes visent à refermer plutôt qu’à ouvrir notre propre connaissance de la société, les sciences sociales ont un rôle démocratique vital à jouer (Connell 2007). Dans le contexte multilingue du Global South, la visibilisation des pratiques langagières et des variétés minorisées d'une part, et l'empowerment via certains types de pratiques langagières de l'autre participent d'un même enjeu de justice sociale. Du point de vue des politiques linguistiques, un chemin possible est l'attribution de droits linguistiques aux langues - pour promouvoir la diversité linguistique et culturelle - ou plutôt aux citoyens comme par exemple le droit à recevoir une éducation décente dans sa langue, dans son environnement langagier habituel, et d'être accueilli à l'hôpital en étant compris. Selon Stroud (2001), même si l'attribution de droits linguistiques permet l'implication de citoyens appartenant à des groupes minorisés, ces concepts excluent de fait les minorités pour lesquelles ils ont été créés. L’alternative selon lui est la citoyenneté linguistique, c’est-à-dire : quand, dans une situation où différentes ressources et pratiques langagières sont disponibles au sein de la société civile, " les locuteurs eux-mêmes exercent une forme de contrôle sur leur langage, décidant quelles langues existent, ce qu'elles signifient et où les problèmes linguistiques sont liés à un ensemble de problèmes sociaux, politiques et de questions d'équité » (ibid. : 353). Le cœur du problème est de savoir, selon lui, comment des identités stigmatisées peuvent gagner en émancipation (être empowered) pour se retrouver dans la situation de partager plus également les ressources symboliques et économiques de la société. Alors que Blommaert (2001) suggère que cette émancipation n'est possible qu'en rendant accessibles les variétés de pouvoir des langues (quelles qu'elles soient) à tous les citoyens, nous avons vu que d'autres voies semblent possibles comme la visibilisation de différentes voix et pratiques minorisées. Parce que «s’intéresser aux inégalités à l’intérieur des langues [on pourrait ajouter s'intéresser à la pluri-accentuation des pratiques langagières], c'est regarder l'économie politique des ressources linguistiques et communicatives dans une société » (ibid : 137), qui plus est si elle est multilingue et que ses citoyens sont plurilingues, les travaux relevant d'approches sociales du langage ont définitivement un rôle démocratique vital à jouer dans le contexte du Global South.

\section{Références bibliographiques}

Alby S. et Léglise I. (2014), "Pratiques et attitudes linguistiques des enseignants. La gestion du plurilinguisme à l'école en Guyane », dans Nocus I., Vernaudon J. et Paia M., (dirs) L'école plurilingue en outre-mer: Apprendre plusieurs langues, plusieurs langues pour apprendre, Rennes, Presses Universitaires de Rennes, p. 245-267.

(2016), «L'éducation bilingue dans le contexte multilingue guyanais : dispositifs cloisonnants et pratiques pédagogiques innovantes » dans Hélot C. et Erfurt, J. (dirs), L'éducation bilingue en France : politiques linguistiques, modèles et pratiques, Limoges, Lambert Lucas, p. 66-86.

Alim H. S. (2004), You Know My Steez: An Ethnographic and Sociolinguistic Study of Styleshifting in a Black American Speech Community, Duke University Press for the American Dialect Society.

(2011), "Global Ill-Literacies: Hip Hop Cultures, Youth Identities, and the Politics of Literacy”, Review of Research in Education 35 (1), p. 120-146.

Auer P. (1999), "From Codeswitching via Language Mixing to Fused Lects: Toward a Dynamic Typology of Bilingual Speech”, The International Journal of Bilingualism 4 (3), p. 309-332. 
Léglise, I. (2017), « Multilinguisme et hétérogénéité des pratiques langagières. Nouveaux chantiers et enjeux du Global South », Langage et Société n¹40-141, 251-266.

Bischoff A. (2003), "Caring for Migrant and Minority Patients in European Hospitals. A Review of Effective Interventions". Ludwig Boltzmann Institute for the Sociology of Health and Medicine, Neuchâtel \& Basel.

Blommaert J. (2001), "The Asmara Declaration as a Sociolinguistic Problem: Reflections on Scholarship and Linguistic Rights” Journal of Sociolinguistics 5 (1), p. 131-142.

Blommaert J. et Backus A. (2011), “Repertoires Revisited: 'Knowing Language’ in Superdiversity” Working Papers in Urban Language and Literacies 67, p. 1-26.

Bourdieu P. et Passeron J-C. (1970), La reproduction: éléments pour une théorie du système d'enseignement. Paris, Editions de Minuit.

Blommaert J. et Rampton B. (2011), “Language and Superdiversity” Diversities 13 (2), p. 1-21.

Boutet J., Fiala P. et Simonin-Grumbach J., (1976) "Sociolinguistique ou sociologie du langage ? " Critique 344, p. 68-85.

Busch B. (2015), "Expanding the Notion of the Linguistic Repertoire: On the Concept of Spracherleben - The Lived Experience of Language" Applied Linguistics, p. 1-20.

Collins J. et Slembrouck S. (2006), “'You Don't Know What They Translate’: Language Contact, Institutional Procedure, and Literacy Practice in Neighborhood Health Clinics in Urban Flanders” Journal of Linguistic Anthropology 16 (2), p. 249-268.

Comaroff J. et Comaroff J. (2012), "Theory from the South: Or, How Euro-America Is Evolving Toward Africa” Anthropological Forum 22 (2), p. 113-131.

Connell R. (2007), Southern Theory: Social Science And The Global Dynamics Of Knowledge. Cambridge; Malden, Polity Press.

Cummins J. (2009), "Fundamental Psycholinguistic and Sociological Principles Underlying Educational Success for Linguistic Minority Students” In Skutnabb-Kangas, R. Phillipson, A. Mohanty, and Panda M., (eds) Social Justice Through Multilingual Education, p. 19-35. Bristol, Multilingual Matters.

Errington J. (2001), “Colonial Linguistics” Annual Review of Anthropology 30 (1), p. 19-39.

Gal S. et Irvine J. (1995), "The Boundaries of Languages and Disciplines : How Ideologies Construct Difference” Social Research 82 (4), p. 967-1001.

García O. (2009a), Bilingual Education in the 21st Century: A Global Perspective. New York, Blackwell / Wiley.

(2009b), "Education, Multilingualism and Translanguaging” In Mohanty A., Panda M., Phillipson R. and Skutnabb-Kangas T. (eds) Multilingual Education For Social Justice: Globalising the Local, p. 140-158. New Delhi, Orient Blackwan.

García O. et Wei L. (2013), Translanguaging: Language, Bilingualism and Education. Palgrave Pivot.

Goury L., Launey M., Lescure O. et Puren L. (2005), "Les langues à la conquête de l'école en Guyane » dans Tupin F. (éd) Ecoles ultramarines, Univers créoles 5, p. 47-65. Paris, Anthropos.

Grenand F. (1982), «Le problème de l'enseignement du français en milieu tribal en Guyane » LENADDOM 66, p. 19-26.

Heller M. et Martin-Jones M. (2001), Voices of Authority: Education and Linguistic Difference. Westport, Ablex.

Hymes D. (1967), "Why Linguistics Needs the Sociologist” Social Research 34 (4), p. 632-647.

Jørgensen J. N., Sif Karrebaek M., Malai Madsen L. et Spindler Moller J. (2011), "Polylanguaging in Superdiversity.” Diversities 13 (2). www.unesco.org/shs/diversities/vol13/issue2/art2.

Kanj M. et Wayne M (2009), "Health Literacy and Health Promotion: Definitions, Concepts and Examples in the Eastern Mediterranean Region”. 7th Global Conference on Health Promotion, Nairobi, Kenya.

Kerfoot C. et Hyltenstam K. (2017), “Introduction: Entanglement and Orders of Visibility”, in Kerfoot C. and Hyltenstam K. (éds), Entangled Discourses: South-North Orders of Visibility, LIEU, Routledge, p. 1-16.

Launey M. (1999), «Les langues de Guyane: des langues régionales pas comme les autres? » dans Clairis C., Costaouec D. et Coyos J-B. (éds), Langues et cultures régionales de France. Etat des lieux, enseignement, politiques, p. 141-159. Paris, L'Harmattan.

Léglise I. (à paraître), « Pratiques langagières plurilingues et frontières de langues », dans Greco L. et Auzanneau M. (dirs), Dessiner les frontières, Lyon, ENS Editions. 
(2007) «Environnement graphique, pratiques et attitudes linguistiques à l’hôpital (St Laurent du Maroni) » dans Léglise I. et Migge B. (dirs), Pratiques et représentations linguistiques en Guyane : regards croisés, Paris, IRD Editions, p. 403-423.

Lüdi G. (1994), « Dénomination médiate et bricolage lexical en situation exolingue » Acquisition et interaction en langue étrangère 3, p. 115-146.

Makoni S. (2011), "Sociolinguistics, Colonial and Postcolonial: An Integrationist Perspective" Language Sciences 33 (4), p.680-688.

Makoni S. et Pennycook A. (2007), Disinventing and Reconstituting Languages. Buffalo, Multilingual Matters.

Migge B. et Léglise I. (2007), "Language and Colonialism. Applied Linguistics in the Context of Creole Communities", in Hellinger M. et Pauwels A. (eds) Language and Communication : Diversity and Change. Handbook of Applied Linguistics 9, p. 297-338.

- 2013, Exploring Language in a Multilingual Context: Variation, Interaction and Ideology in Language Documentation, Cambridge, Cambridge University Press.

Migge B., Léglise I. et Bartens A. (2010), “Creoles in Education. A Discussion of Pertinent Issues”, in Migge B., Léglise I. et Bartens A. (eds) Creoles in Education: An Appraisal of Current Programs and Projects, Amsterdam, John Benjamins, p. 1-30.

Mignolo W. (2009), "Epistemic Disobedience, Independent Thought and Decolonial Freedom" Theory, Culture \& Society 26 (7-8), p. 159-181.

Mondada L. (2012), "L’organisation émergente des ressources multimodales dans l'interaction en lingua franca: entre progressivité et intersubjectivité " Bulletin Suisse de Linguistique Appliquée 95, p. 97-121.

Morrell E. et Duncan-Andrade J. (2004), "What They Do Learn In School: Hip-Hop As a Bridge to Canonical Poetry” In Mahiri J. (ed) What They Don't Learn in School: Literacy in the Lives of Urban Youth, p. 247-268. New York, Peter Lang.

Mudimbe V. (1988), The Invention of Africa: Gnosis, Philosophy, and the Order of Knowledge. Bloomington, Indiana University Press.

Muni Toke V. (2017), "Medical Encounters in a Postcolonial Setting and the Construction of Sociolinguistic Orders of Visibility" In Kerfoot C. and Hyltenstam K. (eds) Entangled Discourses: South-North Orders of Visibility, p. 19-36, Routledge.

Nicolai R. (2007), "Language Contact: A Blind Spot in 'Things Linguistic”" Journal of Language Contact 1: 11-21.

Omoniyi T. (2006), "Hip-Hop through the World Englishes Lens: A Response to Globalization" World Englishes 25 (2), p. 195-208.

Prudent L-F et Schnepel E., (eds) (1993), Creole Movements: The Francophone Orbit. International Journal of the Sociology of Language 102. Walter de Gruyter.

Puren L. (2007), «Contribution à une histoire des politiques linguistiques éducatives mises en œuvre en Guyane française depuis le XIXe siècle » dans Léglise I. et Migge B. (dirs) Pratiques et représentations linguistiques en Guyane, p. 279-298. Paris, IRD Editions.

Rampton B. (2005), Crossing: Language and Ethnicity among Adolescents, Manchester, UK \& Northampton MA, St. Jerome Publishing.

Rojo Martín L. (2015), "The Social Construction of Inequality in and through Interaction in Multilingual Classrooms”, In Numarkee (eds), The Handbook of Classroom Discourse and Interaction, p. 490-505, John Wiley \& Sons, Inc.

Santos de Sousa B. (2011), «Épistémologies du Sud », Etudes rurales 187, p. 21-49.

Skutnabb-Kangas T., Phillipson R., Mohanty A. et Panda M. (2009), Social Justice Through Multilingual Education. Bristol, UK, Multilingual Matters.

Stroud C. (2001), "African Mother-Tongue Programmes and the Politics of Language: Linguistic Citizenship vs. Linguistic Human Rights”, Journal of Multilingual and Multicultural Development 22 (4), p. 339-355.

Thutloa A. et Stroud C., (2013), "Does Active Participation in Health Enhance Health Outcomes and Healthcare Delivery Systems?” Stellenbosch Papers in Linguistics Plus 41, p. 117-123.

Tiouka F. (1984), « Nana iñonoli nana kinipinanon iyombo nana isheman, (Notre terre, nous l’aimons, et nous y tenons). Adresse au Gouvernement et au peuple français ». Premier Congrès des 
Léglise, I. (2017), " Multilinguisme et hétérogénéité des pratiques langagières. Nouveaux chantiers et enjeux du Global South ", Langage et Société n¹40-141, 251-266.

Amérindiens de Guyane française à Awala en présence des autorités administratives locales et de l’Etat, Awala, 9 Décembre.

Vertovec S. (2007), “Super-Diversity and Its Implications” Ethnic and Racial Studies 30 (6), p. 10241054. 